Fetal Diagnosis and Therapy

\title{
Evaluation of Conventional Doppler Fetal Cardiac Function Parameters: E/A Ratios, Outflow Tracts, and Myocardial Performance Index
}

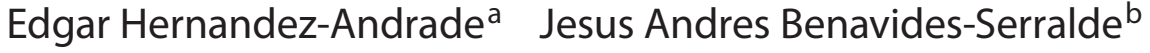 \\ Rogelio Cruz-Martinez ${ }^{c}$ Alec Welsh ${ }^{d}$ Javier Mancilla-Ramirez ${ }^{a}$ \\ ${ }^{a}$ Direction of Clinical Research, and ${ }^{b}$ Department of Maternal Fetal Medicine, National Institute of Perinatology, \\ Mexico City, Mexico; 'Department of Maternal-Fetal Medicine, ICGON, Hospital Clinic-IDIBAPS, \\ University of Barcelona and Centre for Biomedical Research on Rare Diseases (CIBER-ER), Barcelona, Spain; \\ ${ }^{\mathrm{d}}$ Department of Maternal-Fetal Medicine, School of Women's and Children's Health, University of New South Wales, \\ Sydney, N.S.W., Australia
}

\section{Key Words}

Fetal cardiac function - E/A ratios - Outflow tracts ·

Intrauterine growth restriction $\cdot$ Twins

\begin{abstract}
Structural evaluation of the fetal heart is well established. Functional evaluation using pulsed-wave Doppler may also be performed. E/A ratios express the relationship between the maximal velocities of the $E$ and $A$ waveforms of ventricular filling. In normal fetuses, E/A ratios are usually $<1$ but show a constant increment during gestation, mainly related to the increment of the $E$ wave. In intrauterine growth restriction (IUGR) fetuses, E/A ratios are lower compared to values in normally grown fetuses at the same gestational age. Cardiac outflows provide information on the time-velocity integral that, combined with the vessel area, allows calculation of the left and right cardiac outputs. In normal fetuses there is a predominance of the right ventricle (55-60\%) in contributing to the combined cardiac output. In IUGR fetuses this predominance shifts to the left ventricle in order to increase the flow to the upper part of the fetal body and brain. The myocardial performance index (MPI) also provides information on systolic and diastolic cardiac function. The MPI is an early and consistent marker of cardiac dysfunction which be-
\end{abstract}

comes altered in early stages of chronic hypoxia or in cases with cardiac overload such as in twin-to-twin transfusion syndrome.

Copyright $\odot 2012$ S. Karger AG, Basel

\section{Introduction}

Functional fetal cardiac evaluation has been a goal of many fetal medicine researchers for a long time. Whereas ultrasound (US) morphological cardiac examination is now part of routine fetal surveillance, methods of fetal cardiac function measurement have been considered difficult, poorly reproducible, and technically challenging. Analysis of fetal cardiac function might provide important information on the hemodynamic status and cardiovascular adaptation to different perinatal complications. Such information might be useful for the clinical management of pregnancies affected by intrauterine growth restriction (IUGR), complicated monochorionic twin pregnancy, fetal hydrops, diabetes, and fetal arrhythmias. Improvements in US imaging technology may now be making such evaluation possible. A broad range of US techniques have been applied for evaluation of fetal cardiac function including: fundamental image (2D image), 


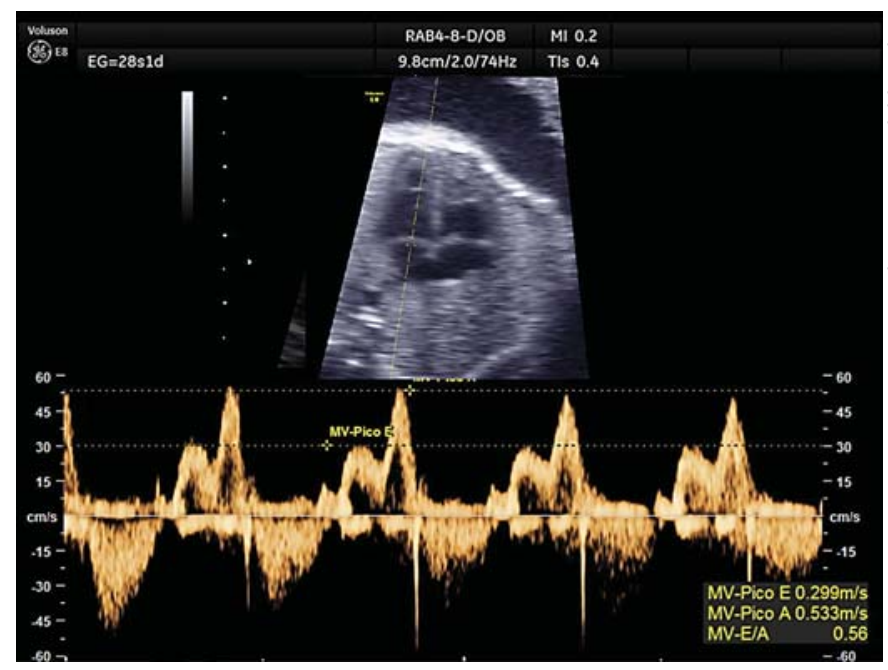

Fig. 1. Mitral E/A waveform.

M-mode, all Doppler modes: directional color, power, spectral conventional, and tissue Doppler, and recently $3 \mathrm{D}$ and $4 \mathrm{D}$ US variants tomographic US imaging (TUI) and spatiotemporal image correlation (STIC). Each of these has its own advantages and limitations.

In this article, we focus on 3 conventional spectral Doppler parameters for estimating fetal cardiac function: the E/A waveforms, the outflow tracts, and the myocardial performance index (MPI). The E/A ratios partially reflect the cardiac preload, whereas the outflow tracts reflect the cardiac afterload, and both are usually evaluated together. The MPI is considered a marker of global cardiac function.

\section{Evaluation of the Fetal E/A Ratios}

Assessment of the fetal diastolic component of the cardiac cycle is performed with spectral Doppler through the atrioventricular (AV) valves. The Doppler sample gate is located just below the AV valves where a biphasic waveform is usually displayed in normal fetus. The first component is known as the $\mathrm{E}$ (early or passive) wave and is related to the process of myocardial relaxation and negative pressure applied by the ventricles. The second component is the A (atrial, active, or late) wave and it represents the atrial contraction during ventricular filling. The ratio is obtained by the division of the peak velocities of the E over the A waveforms (fig. 1,2). The biphasic shape of the E/A waveform can be seen from as early as 9 weeks of gestation as documented by Mäkikallio et al. [1].

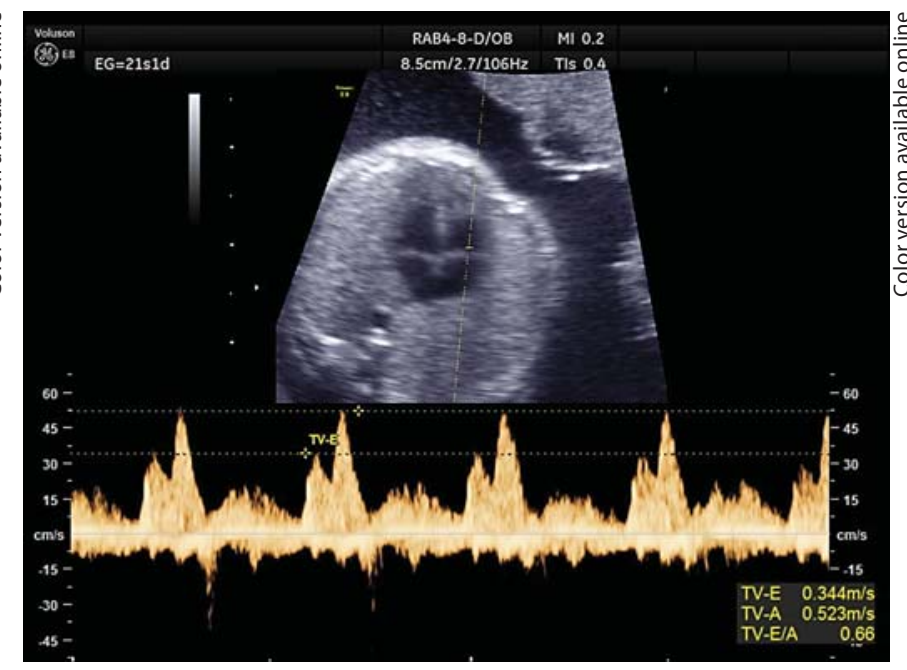

Fig. 2. Tricuspid E/A waveform.

From 10 weeks on, the $\mathrm{E}$ wave is usually smaller than the A wave due to the reduced compliance of the fetal ventricular myocardium. The majority of blood passing to the ventricle is propelled by the atrial contraction, and the $\mathrm{E} / \mathrm{A}$ ratio is usually below 1 . As pregnancy progresses, both $\mathrm{E}$ and $\mathrm{A}$ waves increase, though this is more noticeable in the $\mathrm{E}$ wave, due to a higher ventricular compliance and relaxation of the myocardium [2]. At 16 weeks of gestation, the E wave peak velocity is about $16 \mathrm{~cm} / \mathrm{s}$, then reaching $45-50 \mathrm{~cm} / \mathrm{s}$ at term, whereas during the same period of time the A wave only varies from $30 \mathrm{~cm} / \mathrm{s}$ to $45-50 \mathrm{~cm} / \mathrm{s}$. This progressive increase in $\mathrm{E}$ wave velocity is manifested as a progressive elevation in the E/A ratio in both AV valves throughout gestation [3-5]. After birth, the $\mathrm{E}$ wave is higher than the $\mathrm{A}$ wave; however, the atrial contraction maintains its contribution during the diastolic phase of the cardiac cycle $[6,7]$.

$\mathrm{A}$ reduced $\mathrm{E} / \mathrm{A}$ ratio indicates that the process of ventricular filling depends more on the atrial contraction than on the negative pressure during relaxation. The two main conditions affecting the ratios, chronic hypoxia and cardiac overload, might affect the relaxation process, thus reducing the E/A ratios.

\section{Technical Considerations}

Recordings are obtained at the level of the 4-chamber view of the fetal heart in either a posterior or an anterior apical projection. The interventricular septum must be visualized and aligned at $0^{\circ}$ with the Doppler beam. While E/A ratios are not dramatically affected by the angle of insonation, the image quality improves with a nat- 
ural angle of insonation $<20^{\circ}$. Once the 4 -chamber view and the AV valves are identified, the Doppler sample gate is located immediately distal to either valve [7]. If the angle of insonation is $>20^{\circ}$, it should be manually corrected. It is recommended to keep the Doppler sample gate between 2 and $3 \mathrm{~mm}$ to avoid contamination with artifacts from wall motion and from the outflow tracts. The biphasic nature of the E/A waveform is lost if the Doppler gate is located too deep within the ventricle. Recordings must be performed in the absence of fetal breathing and without maternal and fetal movements.

The pulse repetition frequency should be adjusted until the waveform occupies at least $75 \%$ of the scale and 3-5 symmetrical waveforms are visualized. There is no consensus about the optimal gestational age for performing this evaluation; however, authors start after 20 weeks of gestation. Recent research articles have explored fetal cardiac function at the time of the 11- to $13^{+6}$-week scan [8]. There are some mild differences between both sides of the heart which are constantly observed in all normal pregnancies: the right E/A waveforms have higher velocities and the ratio is slightly lower than that of the left side [9].

Different normal charts during gestation are available in the literature; all presenting similar values [7]. Rizzo et al. [10] reported that, following the technical suggestions previously described, the variation between observers is less than $10 \%$.

\section{Outflow Tracts}

The aortic and pulmonary outflow tracts provide valuable information on the velocity and volume of blood ejected by either ventricle and allow afterload estimation of the cardiac function. Each recording is obtained at the emergence of the aortic or pulmonary arteries just after the semilunar valves. Many measures may be evaluated including: peak systolic velocity, acceleration time, velocity time integral, ejection time (ET), pulsatility, and resistance indices. Both, velocity measurements and vessel area, can be obtained at this anatomical plane, allowing estimation of the right (RCO), left (LCO), and combined cardiac outputs (CCO).

The two outflow tracts represent the peripheral resistance of the vascular system. Each outflow provides blood flow to a different fetal region that might respond differently when a hypoxic insult is present. Blood ejected by the left ventricle is forwarded to the upper part of the fetal body and fetal brain. Changes in the tissue resistance of this region are reflected manly in the left ventricle. Blood

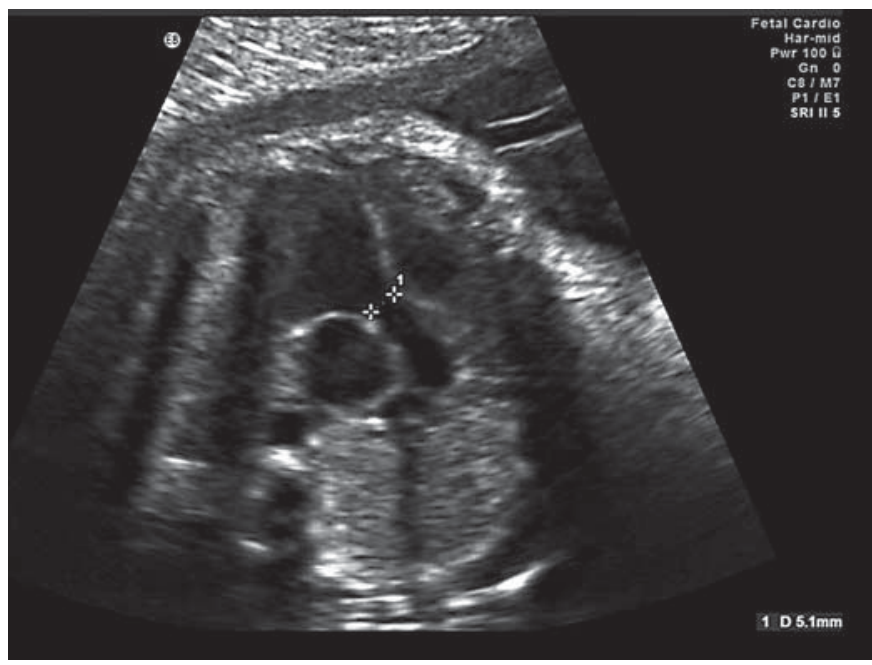

Fig. 3. Left ventricular outflow in a long axis of the left ventricle and measurement of the aortic diameter at the level of the aortic valve.

flow ejected by the right ventricle is mainly forwarded to the lower part of the fetal body, the pulmonary circulation, and the placenta. Increased placental resistance is also mainly expressed in the right ventricle. Normal reference values have been reported by Groenenberg et al. [11] who also showed a mean variation between operators of 5-7\%.

\section{Technical Considerations}

The aortic outflow is evaluated in a long axis of the left ventricle (fig. 3). This plane can be obtained from the 4-chamber view of the fetal heart by rotating the transducer slightly towards the right side where the aorta is visualized as a single vessel arising from the left ventricle in continuity with the interventricular septum and running towards the right side of the heart. The pulmonary outflow tract can be evaluated in either a short or a long axis of the right ventricle (fig. 4). The short axis is obtained from the 4-chamber view of the heart by rotating the transducer slightly towards the left side. The long axis can be obtained from the 4-chamber view of the heart by displacing the transducer slightly towards the fetal head. In the two planes, the pulmonary artery is visualized as a single vessel arising from the ventricle running anteriorly to the left side. In this plane, the semilunar valves can be visualized and the aortic and pulmonary diameters measured. The size of the pulmonary artery is similar or slightly larger than the aorta. The evaluation of both ventricular outflows can be affected by the fetal position and by fetal respiratory or body move- 


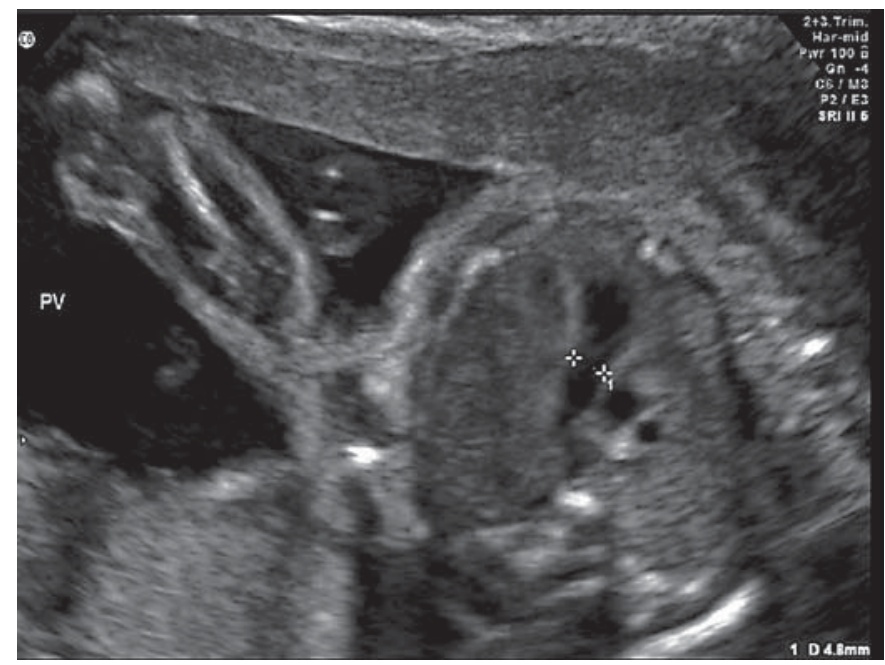

Fig. 4. Right ventricular outflow in a long axis of the right ventricle and measurement of the pulmonary artery diameter at the level of the pulmonary valve.

ments. The time-velocity integral from the aorta or pulmonary outflows is multiplied by the vessel area to estimate the stroke volume (SV). Left and right SV multiplied by the heart rate yield the RCO and LCO which together yield the CCO.

The vessel area is calculated as: $\pi \times(\text { diameter } / 2)^{2}$. The vessel area changes during the cardiac cycle, increasing the variability of the calculations. In order to control this variance, it has been suggested that a minimum of 3 images must be obtained and the mean value used as representative [12]. The error factor induced by a nonreliable diameter calculation is higher at early stages of gestation [13]. Velocity calculation is highly related to the angle of insonation; therefore, an angle as close to zero as possible is necessary to improve the recordings [14]. Normal fetuses show a continuous increment in all cardiac volumes throughout gestation with predominance of the right ventricle, accounting for $55-60 \%$ of the CCO [15].

\section{Technical Limitations}

Doppler waveforms are generally obtained and have a characteristic shape for each outflow tract (fig. 5). The most important information they provide is velocity; thus, the main objective is getting an angle of insonation as close to $0^{\circ}$ as possible. Occasionally, the fetal position increases the technical difficulties in acquiring correct recordings. Nevertheless, an angle of insonation of $30^{\circ}$ with manual correction might be useful for calculation of the LCO or RCO. At an early gestational age, when the

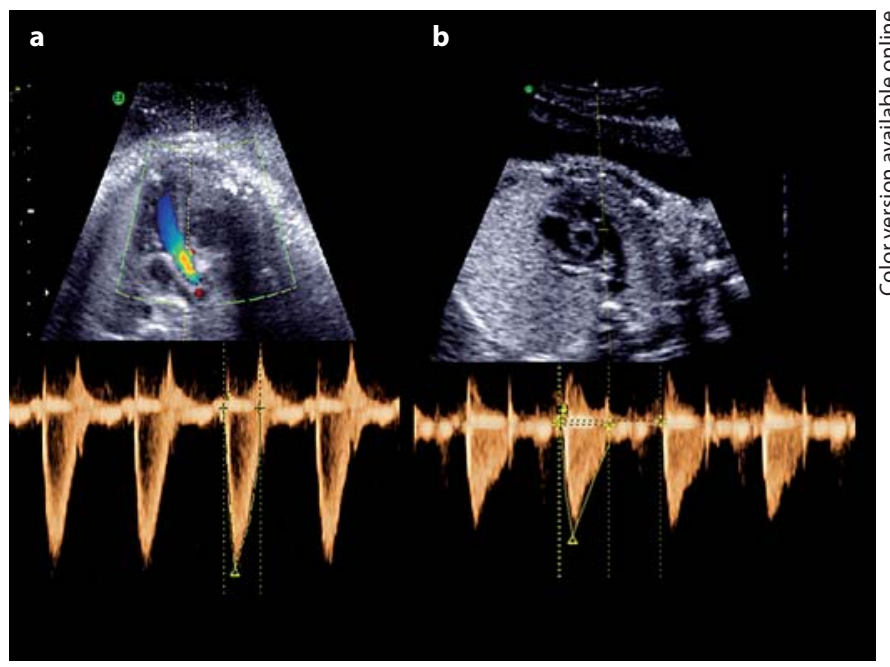

Fig. 5. Aortic and pulmonary (a) arterial Doppler waveforms (b).

heart is small, fetal movements can modify the anatomical location of all cardiac structures affecting the outflow recordings, and fetal breathing movements can also temporally increase the velocity of the outflow tracts.

\section{Clinical Implications of the E/A Ratios and Outflow Tracts}

Visualization of the AV valves and the outflow tracts is mandatory during anatomical evaluation of the fetal heart. If the cardiac anatomy is normal and there are no obstetrical complications, it is not necessary to proceed with the evaluation of cardiac function. In the presence of congenital heart defects affecting the size and emergence of the great vessels, or in the presence of fetal hydrops, IUGR, twin-to-twin transfusion syndrome (TTTS), diabetes, pregnancy-induced hypertension, and fetal arrhythmias, cardiac function might provide important information on fetal hemodynamic adaptation or deterioration. E/A ratios and ventricular outflow tracts are generally evaluated together. In fetal arrhythmias, most of the ventricular filling is dependent on atrial contraction; thus, E/A waveforms change, increasing the A component and reducing the E/A ratio. In fetuses with tachycardia, there is an apparent fusion of both waves, as the time between both components of the waveform is very short [16].

In IUGR fetuses, most reports show a reduction of both E/A ratios compared with normal cohorts $[17,18]$. How- 
ever, Ochi et al. [19] showed an increment in the ratios in early and severely affected IUGR fetuses. In a longitudinal study, Figueras et al. [20] followed 22 IUGR fetuses and reported significantly lower $\mathrm{E} / \mathrm{A}$ ratios in both $\mathrm{AV}$ valves compared to normally grown fetuses with early deterioration of the right $\mathrm{E} / \mathrm{A}$ ratios. For IUGR fetuses, the information offered by E/A ratios alone might be limited but, when combined with other fetal function parameters, it may contribute to understanding the diastolic cardiac period in complicated fetuses. Mäkikallio et al. [21] longitudinally evaluated 75 severely affected IUGR fetuses and compared their fetal cardiac function in relation to the perinatal outcome. They found that those cases with death or neurological damage showed significant differences in almost all prenatal cardiac parameters. Among those parameters, the E/A ratios showed a continuous deterioration, becoming monophasic in the most severely affected IUGR fetuses. In pregnancies complicated by preeclampsia, the ratios seemed not to be affected; however, when preeclampsia and IUGR were both present, E/A ratios showed the same pattern as isolated IUGR [22].

In monochorionic twin pregnancies complicated by TTTS, Van Mieghem et al. [23] showed significant differences in both E/A ratios between donors and recipients before fetoscopic treatment. In uncontrolled diabetic pregnant women, Wong et al. [24] also reported a reduction in the E/A ratios, mainly in the right ventricle, reflecting early fetal cardiac adaptation. In fetuses with premature rupture of membranes and microbial invasion of the amniotic cavity, Romero et al. [25] showed an increment in E/A velocities and ratios in both cardiac ventricles, also indicating early cardiac dysfunction in these fetuses.

Evaluation of cardiac outputs is important in calculating the dominance of the right ventricle during pregnancy and helps to estimate the proportion of blood forwarded to the fetal head and to the superior fetal body [26]; however, in complicated pregnancies, results have been contradictory. In the lamb fetus exposed to acute hypoxia, a reduction in individual cardiac outputs and CCO has been reported [27]. These results have not been reproduced in humans as the cardiac output does not show consistent differences between IUGR and normally grow fetuses. In human fetuses, Figueras et al. [20] showed changes in the maximum velocities of both great arteries in relation to the time of delivery of growth-restricted fetuses. Mäkikallio et al. [28] studied IUGR fetuses in relation the umbilical biochemical markers of cardiac dysfunction and could not identify differences in cardiac output in fetuses showing different levels of these mark-

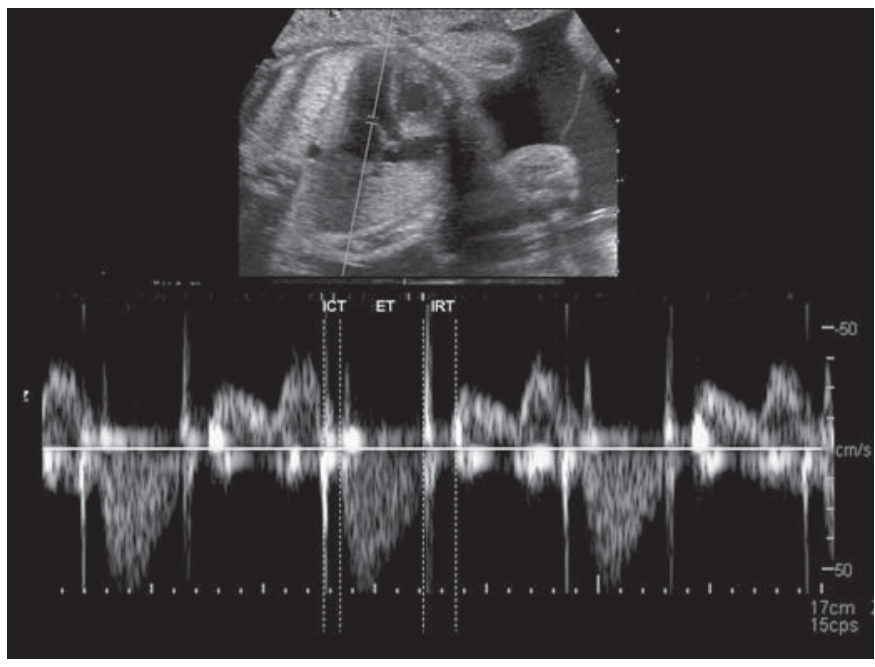

Fig. 6. Myocardial performance index (MPI) measured in the left ventricle. The Doppler waveform displays the clicks of aperture and closure of the mitral and aortic valves. ICT: from the beginning of the closure of the mitral valve until the beginning of the aperture of the aortic valve; ET: from the beginning of the aperture until the beginning of the closure of the aortic valve, and IRT: from the beginning of the closure of the aortic valve until the beginning of the aperture of the mitral valve; MPI = ICT+IRT/ET.

ers, and Bahtiyar and Copel [29] showed that the CCO does not change in IUGR fetuses compared with those normally grown.

\section{MPI (Tei Index)}

The MPI provides information on the different time periods during the systolic phase of the cardiac cycle. MPI can be calculated either by estimating the total isovolumetric time (TIV) over the ET or by estimating the individual isvolumetric contraction (ICT) and relaxation (IRT) times over the ET. The ICT starts when the AV valves close and ends when the semilunar valves open, with a mean duration of $28 \mathrm{~ms}$ (range 23-33). The ET is the period between aperture and closure of the semilunar valves, with a mean value of $175 \mathrm{~ms}$ (range 159-195). The IRT is the period from the closure of the semilunar valves until the aperture of the $\mathrm{AV}$ valves, with a mean value of $34 \mathrm{~ms}$ (range 26-41) [30, 31]. Resulting values for the MPI have been shown to be relatively stable and vary slightly throughout gestation (mean MPI $=0.36$; range $0.28-0.44$ ) [30].

Both ICT and IRT offer information on the first stages of the contractile and relaxation processes of the fetal heart. The ICT expresses the time when the increased pres- 
sure applied by the myocardial contraction opens the semilunar valves. The IRT expresses the time after all blood has been ejected and the semilunar valves are closed, the pressure is reduced, and the process of reuptake of calcium starts [32]. A reduced calcium reuptake reflects a deteriorated cardiac function. In complicated pregnancies, the main parameter of the MPI being affected is the IRT. The ET by itself does not provide robust information on the cardiac status; it is its relationship with the isovulimic times which provides important clinical information. In general, an increased IRT is accompanied by a reduced ET. The ICT is the most stable parameter of the MPI.

\section{Technical Considerations and Limitations for}

Estimation of the MPI

The first reports of the MPI proposed measurement of 2 time periods in different locations and at different moments in the cardiac cycle. The ET is evaluated in the aortic or pulmonary outflow tracts, and the total systolic time is evaluated at the level of the atrial ventricle valves, from the end of the E/A waveform to the beginning of the next E/A waveforms [33]. The systolic time minus the ET represents the total isovolumetric time (IVT), allowing MPI calculation as TIV/ET. This approach had two drawbacks; firstly it is impossible to evaluate the individual isovolumetric times, and secondly small differences in heart rate between the two measurements can affect the final MPI calculation.

To overcome this technical limitation, Friedman et al. [34] showed that all components of the left MPI could be measured in the same waveform, as the aortic and mitral valves are located closer and can be seen in the US screen simultaneously. More recently, different authors [35, 36] refined this estimation, proposing the Doppler clicks of aperture and closure of the valves as landmarks for a better estimation of the time periods. This approach can be applied to the left ventricle throughout gestation, whereas in the right ventricle it can only be applied before 20 weeks of gestation. After 20 weeks, an increase in the distance between the tricuspid and pulmonary valves precludes obtaining both valves in the same Doppler sample gate, requiring right MPI estimation in 2 different locations.

Clear identification of the anatomic landmarks for left MPI estimations has increased its repeatability and widened its application for assessing cardiac function in several perinatal complications. However, some technical aspects must still be considered. Recordings must be performed from the 4-chamber view of the heart with an apical projection of the heart and an angle of insonation below $20^{\circ}$. The transducer is slightly displaced in the cra- nial direction where the mitral and aortic valves are visible. A sample gate of about $3-4 \mathrm{~mm}$ is placed, including the leaflets of both valves. For the US screen display, it is recommended to reduce the gain to exclude noise and artifacts, and to increase the speed of the Doppler baseline to the maximum for clear identification of the anatomical landmarks and time components of the waveform. High wall motion filter (WMF) must be set to avoid recording slow blood movements and to clarify time intervals. Alteration of both angle of insonation and WMF have been found to influence the reproducibility of time interval measurements [37]. Clear valve clicks must be observed in order to correctly place the time cursors. The minimum time that the time cursor can measure must be 2 ms. This estimation should be performed 3 times [30]. US settings must be kept constant for obtaining the 2 individual recordings for the right MPI. The fetal heart rate should be estimated, and if the difference is greater than 10 beats per minute between the 2 recordings, a new estimation must be performed.

Normal reference values and the reproducibility of the left Tei index have been published before [30, 31, 38]. Using the clicks of the valve movements, the reproducibility of the different time components of the left Tei index is more than 0.8 [35]. Comparable results for reproducibility of the right Tei index have been reported despite using two different imaging planes in two different cardiac cycles [39].

\section{Clinical Application of the MPI}

Different authors have proposed different positions of the time cursor for estimation of time intervals including the beginning of valve clicks (original modification of MPI) [35], end of valve click (as physiological representation of the valve click movement) [31], and the peak of valve clicks (as a clearer landmark and to overcome the width of the valve click) [37].

There has been variation in the previously published reference ranges among research groups, and universal agreement for machine settings and phase of the valve click measurement is warranted [37, 40].

The increment in articles including the MPI as part of the fetal evaluation has led to consider this parameter as a reliable early marker of fetal cardiac dysfunction. It probably represents initial stages of cardiac adaptation to different perinatal insults. In IUGR fetuses followed longitudinally, the MPI is one of the earliest parameters affected and remains elevated throughout the different stages of IUGR deterioration [41]. Cruz-Martinez et al. [42] showed that the spatiotemporal cardiac manifesta- 
tion occurs early in the MPI of severely affected IUGR fetuses. Benavides-Serralde et al. [43] evaluated groups of IUGR fetuses classified according to the umbilical artery (UA) Doppler findings and found that the MPI is already affected when the UA waveform still has end-diastolic blood flow. Elevation of the MPI is not a transient sign as it remains higher in complicated cases. It most probably reflects an early adaptive process of the fetal heart to perinatal complications. The association between MPI and biochemical markers of fetal cardiac dysfunction has been reported by Crispi et al. [44].

Hernandez-Andrade et al. [41] showed that the MPI can be considered as a direct parameter of cardiac dysfunction and that in combination with the aortic isthmus and ductus venosus might help to define the deterioration process of severely affected IUGR fetuses. Combination of these parameters might be useful for defining the optimal time of delivery. Its importance as an early and consistent marker of cardiac adaptation has been further explored in diabetic women with poor glycemic control [45]. In TTTS, analysis of cardiac function helps to refine the prognosis of the fetus before laser therapy. Stirnemann et al. [46] showed that $40 \%$ of recipient twins at Quintero stage 1 [47] already show abnormal cardiac function manifested by an altered right MPI. This evaluation was explored further by Papanna et al. [48] who evaluated the MPI before, during, and after laser therapy, reporting a significant increment in the recipient MPI during laser treatment which remained elevated after surgery and became normal only $48 \mathrm{~h}$ after the proce- dure. Other authors have included the MPI in the evaluation of TTTS and propose it as part of the cardiac examination of these fetuses [49, 50]. Letti Müller et al. [51] reported increased values of the MPI in fetuses showing signs of systematic inflammation response syndrome (SIRS). This is in agreement with the results of Romero et al. [25] who also showed abnormal cardiac function in fetuses with PROM and positive microbial cultures in the amniotic fluid. No consistent data is currently available on cases complicated by preeclampsia [52].

\section{Conclusion}

Preload characteristics of the fetal heart are evaluated by the $\mathrm{E} / \mathrm{A}$ ratios in both $\mathrm{AV}$ valves. IUGR fetuses show a reduction in both ratios in relation to the severity of the hypoxic insult. Afterload conditions are expressed by the LCO, RCO, and CCO. IUGR fetuses show a reduction in cardiac outputs and signs of redistribution of the fetal blood by increasing the stroke volume in the left ventricle. There is a predominance of the right ventricle throughout gestation. The MPI is an early and reliable marker of global cardiac dysfunction. These cardiac parameters offer information on the cardiac adaptation to different perinatal complications. Alone, they cannot be used for timing of the delivery, though their combination with other venous and arterial parameters may contribute to defining the fetal condition and to balancing the decision between delivery or continuation of the pregnancy.

\section{References}

1 Mäkikallio K, Jouppila P, Räsänen J: Human fetal cardiac function during the first trimester of pregnancy. Heart 2005;91:334-338.

2 Carceller-Blanchard AM, Fouron JC: Determinants of the Doppler flow velocity profile through the mitral valve of the human fetus. Br Heart J 1993;70:457-460.

-3 Van Splunder P, Stijnen T, Wladimiroff JW: Fetal atrioventricular flow-velocity waveforms and their relation to arterial and venous flow-velocity waveforms at 8 to 20 weeks of gestation. Circulation 1996;94:1372-1378.

4 Reed KL, Sahn DJ, Scagnelli S, Anderson CF, Shenker L: Doppler echocardiographic studies of diastolic function in the human fetal heart: changes during gestation. J Am Coll Cardiol 1986;8:391-395.

5 Tulzer G, Khowasathit P, Gudmundsson S, et al: Diastolic function of the fetal heart during second and third trimester: a prospective longitudinal Doppler echocardiographic study. Eur J Pediatr 1994;153:151-154.
6 Wilson N, Reed K, Allen HD, Marx GR, Goldberg SJ: Doppler echocardiographic observations of pulmonary and transvalvular velocity changes after birth and during the early neonatal period. Am Heart J 1987;113: $750-758$.

7 Rizzo G, Arduini D, Romanini C: Doppler echocardiographic assessment of fetal cardiac function. Ultrsound Obstet Gynecol 1992;2:434-445.

8 Russell NE, Mc Auliffe FM: First-trimester fetal cardiac function. J Ultrasound Med 2008;27:379-383.

-9 Van der Mooren K, Barendregt LG, Wladimiroff JW: Fetal atrioventricular and outflow tract flow velocity waveforms during normal second half of pregnancy. Am J Obstet Gyencol 1991;165:668-674.

10 Rizzo G, Pietropolli A, Capponi A, Cacciatore C, Bufalino L, Arduini D, Romanini C: Echocardiographic studies of the fetal heart. J Perinat Med 1994;22(suppl 1):46-50.
1 Groenenberg IA, Hop WC, Wladimiroff JW: Doppler flow velocity waveforms in the fetal cardiac outflow tract: reproducibility of waveform recording and analysis. Ultrasound Med Biol 1991;17:583-587.

12 Kiserud T, Rasmussen S: How repeat measurements affect the mean diameter of the umbilical vein and the ductus venosus. Ultrasound Obstet Gynecol 1998;11:419-425.

-13 Van Mieghem T, De Koninck P, Steenhaut P, Deprest J: Methods for prenatal assessment of fetal cardiac function. Prenat Diagn 2009; 29:1193-1203.

14 DeVore GR: Assessing fetal cardiac ventricular function. Semin Fetal Neonatal Med 2005; 10:515-541.

15 Kiserud T, Ebbing C, Kessler J, Rasmussen S: Fetal cardiac output, distribution to the placenta and impact of placental compromise. Ultrasound Obstet Gynecol 2006;28:126136 
$\checkmark 16$ Maeno Y, Hirose A, Kanbe T, Hori D: Fetal arrhythmia: prenatal diagnosis and perinatal management. J Obstet Gynaecol Res 2009;35:623-629.

17 Miyake T: Doppler echocardiographic studies of diastolic cardiac function in the human fetal heart. Kurume Med J 2001;48:59-64.

- 18 Severi FM, Rizzo G, Bocchi C, D’Antona D, Verzuri MS, Arduini D: Intrauterine growth retardation and fetal cardiac function. Fetal Diagn Ther 2000;15:8-19.

19 Ochi H, Matsubara K, Kusanagi Y, Furutani K, Katayama T, Ito M: Fetal compromise assessed by Doppler ultrasound of venous flow in pregnancy-induced hypertension. Gynecol Obstet Invest 1999;47:235-238.

-20 Figueras F, Puerto B, Martinez JM, Cararach V, Vanrell JA: Cardiac function monitoring of fetuses with growth restriction. Eur J Obstet Gynecol Reprod Biol 2003;110:159-163.

-21 Mäkikallio K, Räsänen J, Mäkikallio T, Vuolteenaho O, Huhta JC: Human fetal cardiovascular profile score and neonatal outcome in intrauterine growth restriction. Ultrasound Obstet Gynecol 2008;31:48-54.

$\checkmark 22$ Crispi F, Comas M, Hernandez-Andrade E, Eixarxh E, Gomez O, Figueras F, Gratacos E: Does pre-eclampsia influence fetal cardiovascular function in early-onset intrauterine growth restriction? Ultrasound Obstet Gynecol 2009;34:660-665.

-23 Van Mieghem T, Klaritsch P, Doné E, Gucciardo L, Lewi P, Verhaeghe J, Lewi L, Deprest J: Assessment of fetal cardiac function before and after therapy for twin-totwin transfusion syndrome. Am J Obstet Gynecol 2009;200:400.e1-400.e7.

24 Wong SF, Chan FY, Cincotta RB, McIntyre $\mathrm{HD}$, Oats JJ: Cardiac function in fetuses of poorly-controlled pre-gestational diabetic pregnancies - a pilot study. Gynecol Obstet Invest 2003;56:113-116.

25 Romero R, Espinoza J, Gonçalves LF, Gomez R, Medina L, Silva M, Chaiworapongsa T, Yoon BH, Ghezzi F, Lee W, Treadwell M, Berry SM, Maymon E, Mazor M, DeVore G: Fetal cardiac dysfunction in preterm premature rupture of membranes. J Matern Fetal Neonatal Med 2004;16:146-157.

-26 Vimpeli T, Huhtala H, Wilsgaard T, Acharya G: Fetal aortic isthmus blood flow and the fraction of cardiac output distributed to the upper body and brain at 11-20 weeks of gestation. Ultrasound Obstet Gynecol 2009;33: 538-544.

-27 Tchirikov M, Strohner M, Scholz A: Cardiac output and blood flow volume redistribution during acute maternal hypoxia in fetal sheep. J Perinat Med 2010;38:387-392.

-28 Mäkikallio K, Vuolteenaho O, Jouppila P, Räsänen J: Ultrasonographic and biochemical markers of human fetal cardiac dysfunction in placental insufficiency. Circulation 2002;105:2058-2063.

-29 Bahtiyar MO, Copel JA: Cardiac changes in the intrauterine growth-restricted fetus. Semin Perinatol 2008;32:190-193.
30 Hernandez-Andrade E, Figueroa-Diesel H, Kottman C, Illanes S, Arraztoa J, Acosta-Rojas R, Gratacós E: Gestational-age-adjusted reference values for the modified myocardial performance index for evaluation of fetal left cardiac function. Ultrasound Obstet Gynecol 2007;29:321-325.

31 Van Mieghem T, Gucciardo L, Lewi P, Lewi L, Van Schoubroeck D, Devlieger R, De Catte L, Verhaeghe J, Deprest J: Validation of the fetal myocardial performance index in the second and third trimesters of gestation. Ultrasound Obstet Gynecol 2009;33:58-63.

32 Browne VA, Stiffel VM, Pearce WJ, Longo LD, Gilbert RD: Activator calcium and myocardial contractility in fetal sheep exposed to long-term high-altitude hypoxia. Am J Physiol 1997;272:H1196-H1204.

33 Eidem BW, Edwards JM, Cetta F: Quantitative assessment of fetal ventricular function: establishing normal values of the myocardial performance index in the fetus. Echocardiography 2001;18:9-13.

34 Friedman D, Buyon J, Kim M, Glickstein JS: Fetal cardiac function assessed by Doppler myocardial performance index (Tei index). Ultrasound Obstet Gynecol 2003;21:33-36.

35 Hernandez-Andrade E, López-Tenorio J, Figueroa-Diesel H, Sanin-Blair J, Carreras E, Cabero L, Gratacos E: A modified myocardial performance (Tei) index based on the use of valve clicks improves reproducibility of fetal left cardiac function assessment. Ultrasound Obstet Gynecol 2005;26:227-232.

- 36 Raboisson MJ, Bourdages M, Fouron JC: Measuring left ventricular myocardial performance index in fetuses. Am J Cardiol 2003;91:919-921.

37 Meriki N, Izurieta A, Welsh AW: Fetal left modified myocardial performance index: technical refinements in obtaining pulsedDoppler waveforms. Ultrasound Obstet Gynecol. 2012;39:421-429.

38 Meriki N, Welsh A: Development of Australian reference ranges for the left fetal modified myocardial performance index (ModMPI) and the influence of caliper location on valve click measurement. Fetal Diagnosis and Therapy. 2012. In Press.

39 Meriki N, Izurieta A, Welsh A: Demonstration of reproducibility of constituent time in tervals of both right and left fetal modified MPI using pulsed Doppler echocardiography: a short report. Ultrasound Obstet Gynecol. 2012. In Press.

40 Meriki N, Welsh AW: Technical considerations for measurement of the fetal left modified myocardial performance index. Fetal Diagn Ther. 2012; 31: 76-80.

41 Hernandez-Andrade E, Crispi F, BenavidesSerralde JA, Plasencia W, Diesel HF, Eixarch E, Acosta-Rojas R, Figueras F, Nicolaides K, Gratacós E: Contribution of the myocardial performance index and aortic isthmus blood flow index to predicting mortality in preterm growth-restricted fetuses. Ultrasound Obstet Gynecol 2009;34:430-436.
42 Cruz-Martinez R, Figueras F, BenavidesSerralde A, Crispi F, Hernandez-Andrade E, Gratacos E: Sequence of changes in myocardial performance index in relation with aortic isthmus and ductus venosus Doppler in fetuses with early-onset intrauterine growth restriction. Ultrasound Obstet Gynecol 2011;38:179-184.

43 Benavides-Serralde A, Scheier M, CruzMartinez R, Crispi F, Figueras F, Gratacos E, Hernandez-Andrade E: Changes in central and peripheral circulation in intrauterine growth-restricted fetuses at different stages of umbilical artery flow deterioration: new fetal cardiac and brain parameters. Gynecol Obstet Invest 2011;71:274-280.

44 Crispi F, Hernandez-Andrade E, Pelsers MM, Plasencia W, Benavides-Serralde JA, Eixarch E, Le Noble F, Ahmed A, Glatz JF, Nicolaides K, Gratacós E: Cardiac dysfunction and cell damage across clinical stages of severity in growth restricted fetuses. Am J Obstet Gynecol 2008;199:254.e1-254.e8.

-45 Turan S, Turan OM, Miller J, Harman C, Reece EA, Baschat AA: Decreased fetal cardiac performance in the first trimester correlates with hyperglycemia in pre-gestational. Ultrasound Obstet Gynecol 2011;38:325-331.

46 Stirnemann JJ, Mougeot M, Proulx F, Nasr B, Essaoui M, Fouron JC, Ville Y: Profiling fetal cardiac function in twin-twin transfusion syndrome. Ultrasound Obstet Gynecol 2010; $35: 19-27$.

47 Quintero RA, Morales WJ, Allen MH, Bornick PW, Johnson PK, Kruger M: Staging of twin-twin transfusion syndrome. J Perinatol 1999; 19:550-555.

-48 Papanna R, Mann LK, Molina S, Johnson A, Moise KJ: Changes in the recipient fetal Tei index in the peri-operative period after laser photocoagulation of placental anastomoses for twin-twin transfusion syndrome. Prenat Diagn 2011;31:176-180.

49 Van Mieghem T, Klaritsch P, Doné E, Gucciardo L, Lewi P, Verhaeghe J, Lewi L, Deprest J: Assessment of fetal cardiac function before and after therapy for twin-totwin transfusion syndrome. Am J Obstet Gynecol 2009;200:400.e1-400.e7.

50 Rychik J, Tian Z, Bebbington M, Xu F, McCann M, Mann S, Wilson RD, Johnson MP: The twin-twin transfusion syndrome: spectrum of cardiovascular abnormality and development of a cardiovascular score to assess severity of disease. Am J Obstet Gynecol 2007;197:392.e1-392.e8.

51 Letti Müller AL, Barrios Pde M, Kliemann LM, Valério EG, Gasnier R, Magalhães JA: Tei index to assess fetal cardiac performance in fetuses at risk for fetal inflammatory response syndrome. Ultrasound Obstet Gynecol 2010;36:26-31.

52 Api O, Emeksiz MB, Api M, Ugurel V, Unal O: Modified myocardial performance index for evaluation of fetal cardiac function in pre-eclampsia. Ultrasound Obstet Gynecol 2009;33:51-57. 